ACTA UNIVERSITATIS LODZIENSIS

Folia Litteraria Romanica 15, 2020

https://doi.org/10.18778/1505-9065.15.18

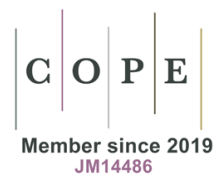

\author{
Anna Żurawska \\ Université Nicolas Copernic \\ (iD) ORCID ID : 0000-0002-5167-6647 \\ zurawska@umk.pl
}

\title{
Du mépris à la dignité : Bakhita de Véronique Olmi
}

\begin{abstract}
RÉSUMÉ
Le roman de Véronique Olmi, Bakhita (2017) retrace l'histoire de sainte Joséphine Bakhita en démontrant le processus qui va du mépris, de l'humiliation, de l'animalisation à l'acquisition de la dignité, voire à la sainteté. Le texte met l'accent non pas sur la dégradation des sentiments et de l'être humain, mais il montre comment dépasser les limites imposées par le mépris des autres et atteindre la plénitude. L'objectif du présent article sera, dans un premier temps, d'étudier des manifestations du mépris et des moyens littéraires qui permettent de l'exprimer. Dans un deuxième temps, l'analyse se centrera sur le processus qui va du mépris vers la plénitude, dans l'acception que lui donne Charles Taylor. Enfin, il sera aussi intéressant de poser la question sur les raisons du succès de ce roman « hagiographique » en France laïque.
\end{abstract}

MOTS-CLÉS - Bakhita, esclavage, mépris, plénitude, dignité, la beauté intérieure, sécularisation

\section{"From Disdain to Dignity - Bakhita by Véronique Olmi"}

\begin{abstract}
SUMMARY
Véronique Olmi's novel entitled Bakhita (2017), telling the story of St. Josephine Bakhita, shows the process of achieving dignity, or even sanctity, through overcoming disdain, humiliation, and animalization. However, it is not the degradation of feelings and a human being that is emphasized in Olmi's novel but the possibility of overcoming the condition of a disdained person in order to enjoy freedom and fullness. The aim of the article is therefore to explore the manifestations of disdain in the analyzed text and the use of rhetorical devices applied to express contempt. The article also examines the process leading from disdain to fullness as defined by Charles Taylor, as well as the reasons for the popularity of the novel in France, regarded as the most laic country in Europe.
\end{abstract}

KEYWORDS - Bakhita, slavery, disdain, the place of fullness, dignity, inner beauty, secularization 
Passé à la troisième sélection du Prix Goncourt 2017, le treizième roman de Véronique Olmi, Bakhita, était très proche de recevoir le prix. Bien qu'il ait cédé la première place du palmarès à Éric Vuillard et à son Ordre du jour, le texte d'Olmi a été récompensé par plusieurs autres prix ${ }^{1}$. Son succès est confirmé aussi par le nombre d'exemplaires vendus ${ }^{2}$ et des traductions du texte en différentes langues. Le roman a été aussi traduit en polonais par Katarzyna Marczewska ${ }^{3}$ et publié par la maison d'édition Wydawnictwo Literackie en octobre 2018.

Le texte, partagé en deux parties portant des titres significatifs : «De l'esclavage à la liberté » et « De la liberté à la sainteté », retrace l'histoire de Bakhita en démontrant le processus qui va du mépris, de l'humiliation, de l'animalisation à l'acquisition de la dignité, voire à la sainteté. La narration met l'accent non pas sur la dégradation de l'être humain, comme c'est le cas du fameux roman d'Antonio Moravia Il disprezzo, adapté au cinéma par Jean-Luc Godard, mais il montre comment dépasser les limites imposées par le mépris des autres et vivre la plénitude.

La problématique du présent article consistera donc à analyser ce processus de cheminement du mépris vers la dignité et la plénitude. Celle-ci va être comprise en accord avec la conception de Charles Taylor qui constate que « [1]e pouvoir d'accéder à la plénitude part de l'intérieur $»^{4}$. Il explique aussi qu'au moment où nous atteignons « le lieu de plénitude»,

la vie nous apparaît plus pleine, plus riche, plus profonde, plus digne d'intérêt, plus admirable, plus fidèle à ce qu'elle doit être. C'est peut-être un lieu où se déploie une puissance : nous faisons souvent ici l'expérience de quelque chose de profondément émouvant, susceptible de nous inspirer. [...] nous ressentons une puissante intuition de ce que serait la plénitude - paix, complétude... - si nous pouvions y accéder ou si nous étions capables d'agir à ce niveau, c'est-à-dire avec intégrité, générosité, abandon ou oubli de soi ${ }^{5}$.

Afin de comprendre l'arrivée du personnage éponyme à ce « lieu de plénitude » défini par Taylor, nous étudierons, d'abord, les manifestations du mépris et les moyens littéraires qui permettent de l'exprimer, puis, le processus intérieur de construction de la dignité. Finalement, il sera aussi intéressant de poser la question sur des raisons du succès de ce roman « hagiographique » en France laïque.

1 Prix du roman FNAC 2017 ; Choix Goncourt de l'Orient 2017 ; Choix Goncourt de la Serbie 2018 ; Prix des lecteurs des écrivains du Sud ; Grand Prix des Blogueurs 2017 ; Finaliste Prix Goncourt : le choix polonais 2017 ; Finaliste Prix Goncourt des lycéens 2017 ; Finaliste Prix Femina 2017.

246500 exemplaires. Cf. N. Carreau et G. P., Prix Goncourt : pourquoi notre spécialiste voit "Bakhita », de Véronique Olmi, gagner ; URL : http://www.europe1.fr/culture/prix-goncourtvoici-notre-favori-3482600; consulté le 2.09.2018.

3 Le Premier amour (2010), un autre roman d'Olmi, a été aussi traduit en polonais, par A. SylwestrzakWszelaki, et publié en 2011 par Wydawnictwo Otwarte sous le titre Czekam na Ciebie.

4 Ch. Taylor, L'Âge séculier, trad. P. Savidan, Paris, Seuil, 2011, p. 25.

5 Ibid., p. 18-19. 


\section{Biographie de Bakhita et genèse du roman}

Le roman, qu'il est plus convenu d'appeler biographie romancée ${ }^{6}$, retrace l'histoire de Bakhita, ce qui témoigne du travail de chercheur qu'a dû faire l'écrivaine ${ }^{7}$. Mais la narration à la troisième personne, qui dans ce cas-là est linéaire, se focalise aussi sur l'intériorité du personnage : ses pensées, ses sentiments, ce qui constitue la part de fiction. La narration n'est pourtant pas neutre, la sympathie et l'admiration du narrateur pour la protagoniste étant manifestes.

L'histoire de Bakhita serait inconcevable s'il ne s'agissait pas d'un personnage réel. Tout comme sa grande sœur quelques années plus tôt, elle est enlevée par les marchands d'esclaves à l'âge de sept ans (probablement vers 1875, ni l'âge ni la date exacts ne sont connus) de son village natal dans l'ouest du Soudan (il est impossible de préciser le lieu de sa naissance). Vendue plusieurs fois à divers maîtres, elle souffre des tortures, des humiliations, un viol, des châtiments, la marche épuisante à travers le désert, etc. Elle est finalement achetée par le consul italien, Calisto Legnani, qui consent à l'emmener en Vénétie où elle est domestique avant d'embrasser la religion chrétienne et d'intégrer le couvent pour devenir religieuse. Pourtant, avec l'arrivée en Europe, la souffrance ne finit pas, elle change de nature. Cette fois-ci, elle doit faire face au racisme, aux deux guerres mondiales et à l'usage de son image dans la propagande coloniale de Mussolini. « Le Soudan, l'Italie, c'est la même beauté et le même mal $»^{8}$, conclura le narrateur. De plus, le tragique de Bakhita consiste dans le fait qu'elle n'arrive pas à parler une langue : à part quelques paroles, elle a oublié sa langue maternelle, y compris son prénom (Bakhita qui veut dire « la chanceuse », c'est le surnom que lui ont donné les marchands d'esclaves, par contre Joséphine / Giuseppina est son prénom européen de baptême ${ }^{9}$ ), elle connaît quelques mots de l'arabe et de différents dialectes africains en fonction des maîtres chez qui elle travaillait, finalement, elle apprend un peu le dialecte vénitien. Elle n'arrive donc pas à s'exprimer librement dans une langue et à communiquer son

6 Olmi constate pourtant : «Je voulais écrire un roman, pas une biographie, alors j’ai travaillé davantage le langage, plutôt que les archives, j'ai essayé d'être au plus près d'elle [de Bakhita] ». L'interview avec V. Olmi par V. Trierweiler, « Véronique Olmi plonge dans le mystère. Bakhita », Paris Match, 03.09.2017 ; URL : https://www.parismatch.com/Culture/Livres/Veronique-Olmiplonge-dans-le-mystere-Bakhita-1339076; consulté le 2.09.2018.

7 Olmi s'est surtout inspirée de Storia meravigliosa della vita (1931), l'histoire qu'Ida Zanolini a écrite à partir des conversations avec Bakhita. Mais le début du XXI ${ }^{\mathrm{e}}$ siècle est aussi riche en autres ouvrages sur Bakhita, entre autres deux biographies écrites en 2015 et 2019 par H. Roullet (voir la bibliographie) ou le film Bakhita tourné par G. Campiotti en 2009.

8 V. Olmi, Bakhita, Paris, Albin Michel, 2017, p. 334. Désormais, les citations du roman seront suivies de la lettre $B$. et du numéro de page entre parenthèses.

9 Évocatrice est dans ce contexte la citation de Si c'est un homme de Primo Levi, mise en exergue : « Ils nous enlèveront jusqu'à notre nom : et si nous voulons le conserver, nous devrons trouver en nous la force nécessaire pour que derrière ce nom, quelque chose de nous, de ce que nous étions, subsiste $» ; B ., 9$. 
histoire douloureuse. L'incipit du roman insiste sur cela : « Elle ne sait pas comment elle s'appelle. Elle ne sait pas en quelle langue sont ses rêves » $(B ., 13)$. Elle meurt en 1947 et est canonisée en 2000 par Jean-Paul II ${ }^{10}$.

Véronique Olmi affirme avoir découvert Bakhita par hasard en visitant une église à Langeais en Touraine où se trouvait son portrait avec quelques informations biographiques ${ }^{11}$. Cela l'a inspirée à entreprendre des recherches et à écrire son roman à l'origine duquel il y a un mystère, une question articulée par Olmi dans une des interviews : «[c]omment tant de bonté peut naître de tant de souffrance ? $»^{12}$. Elle ajoute que le vrai «questionnement, c'était : quelle est l'intériorité de cet être exceptionnel ? ${ }^{13}$.

\section{Le mépris}

Le dictionnaire de la langue française, Le Petit Robert, propose quatre définitions du mépris, entre autres : « fait de considérer comme indigne d'attention [...], indifférence, dédain »; « sentiment par lequel on s'élève au-dessus de » ou encore « sentiment par lequel on considère qqn comme indigne d'estime, comme moralement condamnable $»^{14}$.

Dans la Psychologie des émotions, on lit que le mépris est une des plus subtiles émotions sociales pareillement à la honte, le sentiment de culpabilité, la jalousie, etc. ${ }^{15}$, qui est communiquée par les paroles ou comportements agressifs (insultes, gestes violents, etc.). Le psychologue Richard Wiseman explique que « la vie manque de sens quand le mépris, soit-il dirigé vers soi ou vers une autre personne, parvient à détruire la confiance en soi et la curiosité d'approfondir la connaissance et l'amour des autres $\rangle^{16}$.

Dans le contexte de notre étude, il faut prendre en compte aussi la perspective spirituelle ce qui paraît d'autant plus pertinent que dans sa conception de plénitude, Taylor marie l'aspect spirituel aux sentiments, donc à une expérience de nature

${ }^{10}$ Les informations biographiques viennent du Dictionnaire biographiques des chrétiens d'Afrique ; URL : https://dacb.org/fr/stories/sudan/bakhita-josephine2 ; consulté le 2.09.2018.

${ }^{11}$ Interrogée sur cette découverte, elle le répète presque dans chaque interview, ex. "Bakhita » de Véronique Olmi ou le parcours d'une esclave devenue Sainte, TV5 MONDE DIRECT ; URL : https:/www.youtube.com/watch?v=aLk7YKAhcI0 ; consulté le 2.09.2018.

${ }^{12}$ V. Trierweiler, «Véronique Olmi plonge... », op. cit.

${ }^{13}$ La Grande Librairie : «Bakhita » racontée par Véronique Olmi ; URL : https://www.youtube.com/ watch?v=6A_EdcyHNbA; consulté le 2.09.2018.

${ }^{14}$ Le Nouveau Petit Robert, sous la dir. de J. Rey-Debove, A. Rey, Paris, Dictionnaires Le Robert, p. 1557.

${ }^{15}$ M. Lewis, J. M. Haviland-Jones, Psychologia emocji, trad. M. Kacmajor et al., Gdańsk, Gdańskie Wydawnictwo Psychologiczne, 2005, p. 193.

${ }^{16}$ Cité d'après La Confiance est le meilleur vaccin contre le mépris ; URL : https://nospensees.fr/ confiance-meilleur-vaccin-contre-mepris/ ; consulté le 25.09.2018. 
émotionnelle. Le philosophe n'évoque dans ce cas que des émotions à connotation positive, mais le vécu de l'expérience spirituelle peut être accompagné tout aussi bien d'angoisse, de mélancolie ou de culpabilité ${ }^{17}$. Il convient ainsi de rappeler que dans la Bible, le mépris apparaît dans le monde avec le péché originel et prend la forme encore plus concrète dans la scène du fratricide commis par Caïn. Le texte d'Olmi semble dialoguer avec ce contexte vétérotestamentaire en explorant la symbolique biblique (arbre, insistance, honte, crime) : « [1]'homme insiste. Elle regarde l'arbre qu'il lui [à Bakhita] demande d'atteindre. Elle ne sait pas pourquoi, mais elle le fait. Elle va vers l'arbre. L'homme [...] sort un poignard et le met contre sa gorge...» $(B ., 29)$. Véronique Olmi conclut que Bakhita, kidnappée près d'un bananier, ressent la honte d'être nue, «à partir de là, son corps, sa beauté sera toujours une offense $»^{18}$.

Les définitions évoquées ci-dessus, soient-elles linguistiques, psychologiques ou bibliques, considèrent le mépris aussi bien du point de vue de la personne méprisante que celui de l'être méprisé, en soulignant soit la nature de cette émotion et la manière de l'exprimer, soit le manque de confiance en soi (et dans les autres) qui est le résultat direct du mépris. Dans ce contexte, il convient de poser la question sur les moyens romanesques qui permettent de représenter le mépris, ainsi que de se demander dans quelle mesure le texte correspond aux définitions données et quelle est la part d'une nouvelle compréhension de cette notion.

\section{Représentations romanesques du mépris}

Le mépris envers les esclaves se traduit par quelques mécanismes tels que la réification et l'animalisation de l'être humain, bref, la privation de son statut de personne. Le roman en rend compte en décrivant de terribles conditions de vie des esclaves et en mettant l'accent sur le sentiment de supériorité qui caractérise les trafiquants d'esclaves et les maîtres, donc le sentiment «par lequel on s'élève au-dessus de $»^{19}$ l'autre.

Premièrement, du point de vue formel, le développement des champs lexicaux précis a contribué à illustrer la réification des prisonniers, y compris Bakhita. D'un côté, il s'agit du vocabulaire qui réduit les personnes au statut d'un objet ( « la marchandise», $B ., 49$; « immobiles et muettes, comme les tapis et les coussins autour, elles attendent un ordre », B., 143). Mais de l'autre, tout le lexique lié au corps humain ôte la personne de ses autres dimensions : intellectuelle, spirituelle, émotionnelle, comme l'illustre la description de la vente des esclaves : «c'est chaque

\footnotetext{
${ }^{17}$ Cf. Ch. Taylor, La Diversité de l'expérience religieuse aujourd'hui. William James revisité, trad. J.-A. Billard, Québec, Éditions Bellarmin, 2003.

${ }^{18}$ La Grande Librairie, op. cit.

19 J. Rey-Debove, A. Rey (dir.), op. cit., p. 1557.
} 
fois le même processus : avant d'acheter ils vérifient les dents, les yeux, la peau, dedans, dehors, les muscles, les os $»(B ., 43)$. À plusieurs reprises, la narration insiste sur la nudité de ce corps $(B ., 29,38)$, sa saleté $(B ., 33)$ et ses réactions physiologiques (la faim, la soif, les maladies, $B ., 44$ ).

Le dernier champ lexical, qu'il vaut la peine d'évoquer, est le vocabulaire lié au monde animal. La nature joue dans ce roman deux fonctions différentes : l'une, qui sera l'objet de l'étude ultérieure, donne naissance au mysticisme de Bakhita, l'autre sert à illustrer l'animalisation de l'homme : « On lui [à Bakhita] lance un bâton pour qu'elle coure et le ramène, au début elle ne comprend pas. Elle ne va pas le chercher. On la gifle et on recommence. Elle court » $(B ., 36)$. Puis, la coexistence avec des animaux de pire catégorie : vermine, rats, etc., renforce encore cette image (cf. B., 33). Mais l'effet est aussi obtenu grâce à des figures de style consciemment utilisées par le narrateur telles que métaphores ou comparaisons : «Elle crie comme un animal abandonné » $(B ., 35)$.

Deuxièmement, le mépris et l'angoisse sont exprimés non seulement au niveau lexical, mais aussi syntaxique d'abord grâce au recours à des phrases très courtes, souvent nominales :

Et un matin, on les expose sur le grand marché. C'est un jour attendu et redouté. La mise en vente. [...] On la désigne, on la désentrave, elle s'avance, et elle fait ce qu'on lui demande de faire. Comme d'habitude. De face. De dos. Vite. Lentement. Les yeux baissés. La tête renversée. Calme et sans expression. Patiente et obéissante (B., 97).

Parfois, cette syntaxe imite aussi les ordres donnés par les trafiquants ou bien la marche des esclaves, le rythme répétitif de leurs pas et de leur respiration, le bruit des chaînes : "Ils sont attachés les uns aux autres. Les hommes devant. Trois. Les chaînes autour du cou, reliées au cou des deux autres. Les femmes derrière. Trois. Les chaînes autour du cou. Reliées au cou des deux autres » $(B ., 38)$. Ce choix syntaxique recrée la terreur, mais vu qu'il est d'une grande simplicitée ${ }^{20}$, peut-être, il vise aussi à raconter l'histoire de Bakhita sans pathos et avec le respect pour le personnage qui consiste à ne pas dire trop.

À la lumière de cette brève analyse, les définitions évoquées ci-dessus, où le mépris est nécessairement motivé, semblent insuffisantes par le fait de ne pas prendre en compte le caractère gratuit de ce sentiment.

${ }^{20}$ Pourtant, cette simplicité est critiquée par Grégoire Leménager qui reproche à la narration d'être « très factuelle, extrêmement linéaire ». Selon le critique, Olmi n'a pas réussi à exploiter pleinement le potentiel de la biographie de Bakhita. «Le clash culture : faut-il lire Bakhita de Véronique Olmi ? », Le Figaro.fr 02.12.2017 ; URL : http://www.lefigaro.fr/livres/2017/12/02/0300520171202ARTFIG00012-le-clash-culture-faut-il-lire-bakhita-de-veronique-olmi.php ; consulté le 25.09.2018. 


\section{Du mépris vers la plénitude}

L'approche psychologique du mépris fait aussi défaut dans le cas de Bakhita. Wiseman affirme que le résultat direct du mépris est la perte de « confiance en soi et [de] curiosité d'approfondir la connaissance et l'amour des autres $\gg^{21}$. Or, l'exemple de Bakhita contredit cette constatation, tout en montrant d'un côté sa dignité et de l'autre, son dévouement, son amour, son attention envers les autres aussi bien durant son esclavage que pendant sa vie de religieuse. En effet, ce qui intéresse le plus Véronique Olmi, c'est la réflexion sur la construction de l'humain sur le fondement de l'inhumain. L'écrivaine répète, à plusieurs reprises, qu'elle ne comprenait pas « comment une enfant sans enfance pouvait grandir en humanité ${ }^{22}$, « [c]omment fait-on quand on n'a pas de souvenirs, de témoins, de photos, de dessins, de lieux ? Comment fait-on pour se construire, quelle est cette force d'âme ? [...] Il fallait faire preuve d'une grande résilience pour se sauver psychiquement $\gg^{23}$, elle ajoute que « cette résilience qui était plus qu'une résilience, c'était un être de bonté extrême [...] elle était un être tournée vers les autres et moi, ce qui m'a intéressée, c'est l'intériorité... $»^{24}$.

Le questionnement de l'écrivaine est légitime aussi à la lumière de l'analyse des conditions de la formation du sentiment de la dignité qui « commence [à se former, AŻ] à la première étape du processus de développement par le moyen de l'imitation et de l'identification, surtout avec les parents et les héros, et aussi de l'interaction avec le groupe $»^{25}$.

Dans le cas de Bahkita, il semble qu'aucune de ces conditions n'ait été respectée ${ }^{26}$. Il est donc intéressant de voir comment l'écrivaine essaie de répondre aux questions qui l'ont interpellée et inspirée à écrire le roman. Sans prétendre à être exhaustive, la liste qu'il est possible de dresser à partir du texte peut contenir quelques conditions qui ont permis à Bakhita de se protéger contre le mépris et de reconstruire sa dignité. D'abord, ce sont des souvenirs d'avant l'enlèvement. Parfois, ils sont articulés par le narrateur comme s'il s'agissait des visions presque réelles (B., p. 157), parfois, ils apparaissent sous forme d'une sensation sonore. Puis, de pair avec les souvenirs va son imagination qui lui permet de se créer un univers à elle où elle peut séjourner quand la réalité devient insupportable :

${ }^{21}$ Cité d'après La Confiance est le meilleur vaccin, op. cit.

${ }^{22}$ La Grande Librairie, op. cit.

${ }^{23}$ V. Trierweiler, «Véronique Olmi plonge... », op. cit.

${ }^{24}$ "Bakhita » de Véronique Olmi ou le parcours d'une esclave devenue Sainte, TV5 MONDE DIRECT, op. cit.

${ }^{25}$ L. Bieńkowski et al., Encyklopedia katolicka, vol. V, Lublin, Katolicki Uniwersytet Lubelski, p. 1231. La traduction du polonais faite par l'auteure de l'article.

${ }^{26}$ Pourtant, selon Véronique Olmi, elle a dû être très aimée avant l'enlèvement ce qui lui a donné de la force. $C f$. « Bakhita » de Véronique Olmi ou le parcours d'une esclave devenue Sainte, TV5 MONDE DIRECT, op. cit. 
Alors elle trouve un autre moyen de se sauver. Les histoires...[...] Elle imagine parfois que les tout-petits l'écoutent, elle revoit leurs yeux pleins de peur et d'espérance, elle commence l'histoire et elle ne finit jamais [...] elle plonge dans le monde d'avant, où elle entend les cris le soir pour ramener les troupeaux. Les appels de sa mère pour venir manger (B., p. 35).

Son imagination a également le pouvoir d'inverser le processus de l'animalisation. Or, elle procède à la personnification des animaux en leur donnant un nom et se réjouissant de leur existence. Par cela, elle redonne le caractère humain à son entourage : « elle change les scorpions, les rats et les fourmis en personnes aimées, elle les nomme, elle les regarde vivre. Un temps, cette autre réalité la sauve de la mort » (B., p. 35).

Elle est aussi très empathique envers d'autres esclaves et entretient des relations avec eux, surtout avec des filles encore plus petites qu'elle, dont elle prend soin et qu'elle entoure d'amour et de tendresse : « elles dorment [Bakhita et son amie Binah] en se tenant la main. Bakhita sent alors une force insoupçonnée, un courant puissant, et cela aussi est nouveau : partager avec une inconnue l'amour que l'on ne peut plus offrir à ceux qui nous manquent » $(B .$, p. 46).

La nature est aussi source de beauté et de mystère ressentis par Bakhita. Comme le remarque Olmi «elle [Bakhita] a été [...] sauvée par la beauté du monde, parce qu'elle a toujours su la trouver $»^{27}$. La capacité d'admirer la nature (ce qui témoigne aussi de sa grande sensibilité) lui vient, peut-être, de la religion animiste de son village $^{28}$, mais cette attitude contemplative est aussi caractéristique des mystiques chrétiens (B., p. 40-41, 54 sqq.) : «Elle entend [...] le silence qui recouvre tout. [...] Bakhita pense qu'el Paron a créé la nuit pour le repos des hommes et des bêtes, et aussi pour rien. Pour la beauté » (B., p. 349-350). Sa vie de religieuse semble une naturelle continuation de l'approfondissement de son sentiment de mystère, de quelque chose qui est plus grand que le mal qu'elle subit. Bakhita ne reçoit aucune éducation régulière, mais sa sagesse et sa connaissance du monde sont d'un autre ordre, celui du cœur. Dans son encyclique Fides et ratio, Jean-Paul II rappelle que : «Les connaissances fondamentales découlent de l'émerveillement suscité en lui [en l'homme, AŻ] par la contemplation de la création : l'être humain est frappé d'admiration en découvrant qu'il est inséré dans le monde, en relation avec d'autres êtres semblables à lui dont il partage la destinée $»^{29}$. Bakhita, qui «a traversé le chaos du monde $»^{30}$, n'a nullement perdu cette capacité d'émerveillement, de contemplation, de compréhension qu'elle fait partie de l'univers, sa relation avec

${ }^{27}$ La Grande Librairie, op. cit.

${ }^{28}$ «La terre lui a parlé, la terre sacrée qu'honorent les gens de sa tribu s'est adressée à elle». V. Olmi, op. cit., p. 146.

${ }^{29}$ Jean-Paul II, Lettre Encyclique Fides et ratio, p. 3 ; URL : https://www.eleves.ens.fr/aumonerie/ talatex/fides ratio/tex/fides ratio.pdf; consulté le 10.04.2019.

${ }^{30}$ La Grande Librairie, op. cit. 
Dieu, telle qu'elle a été présentée dans le roman, s'appuie sur une forte liaison avec «d'autres êtres semblables »" à elle et avec la nature.

Véronique Olmi affirme vouloir respecter l'intimité de la vie intérieure de Bakhita $^{32}$ et dans le roman rares sont des descriptions de son arrivée à la foi et de sa relation avec Dieu, mais le texte insiste sur une certaine nostalgie que le personnage ressent et qui semble s'apaiser avec l'entrée au couvent.

Quelle que soit la manière d'approcher les sources profondes de sa force intérieure, le mystère persiste. Dans le roman, la protagoniste ne vit pas une illumination soudaine qui change sa perception de la vie (comme c'est p. ex. le cas de Bede Griffiths cité par Taylor qui explique les sentiments possibles accompagnant l'arrivée à la plénitude). Bakhita semble construire lentement sa dignité sur l'acceptation de son sort, l'oubli de soi et sur le sentiment qu'il y a quelque chose qui transcende la souffrance, le chaos du monde où elle vit, bref, sa condition humaine.

\section{Réception du roman}

Reste encore la question du succès du roman, qui présente la vie d'une sainte, dans la société française qui est pourtant considérée comme l'une des plus sécularisées de l'Europe ? La première explication de la réussite de Bakhita, qui est arrivé en finale du Prix Goncourt 2017, est l'intérêt qu'on porte de nos jours à la question d'esclavage et de liberté ${ }^{33}$, donc ce qui intéresserait le plus les lecteurs français, c'est surtout la première partie du roman décrivant la situation des esclaves. Véronique Olmi y consent et élargit encore l'horizon de ce problème, elle constate :

Bakhita m'a amenée à une conscience plus aiguë de l'esclavage moderne, réparti dans tous les milieux. Je cherche quoi faire de cette prise de conscience. À Paris et ailleurs, il y a beaucoup de petites Bakhita. J'ai écrit ce livre au moment des attentats du Bataclan et de Nice, je sais les phrases qui parlent d'aujourd'hui à travers hier ${ }^{34}$.

Pourtant, cette question ne paraît pas suffisante pour la compréhension du succès de cette hagiographie contemporaine, d'autant plus qu'Olmi, elle-même, ne privilégie pas seulement l'esclavage, mais aussi la force intérieure de la personne qui en sort en conservant sa dignité, elle le répète à plusieurs reprises : « Elle avait une force d'insoumission intérieure $»^{35}$.

\footnotetext{
${ }^{31}$ Jean-Paul II, op. cit., p. 3.

${ }^{32} C f$. «Bakhita » de Véronique Olmi ou le parcours d'une esclave devenue Sainte, TV5 MONDE DIRECT, op. cit.

${ }^{33}$ Cf. Z. N. Hurston, Barracoon: The Story of the Last 'Black Cargo', Amistad Press, New York, 2018.

${ }^{34}$ V. Trierweiler, «Véronique Olmi plonge... », op. cit.

${ }^{35}$ Ibid.
} 
Les raisons du succès de ce roman paraissent plus complexes. Charles Taylor constate que nous vivons dans la société où domine " un humanisme purement autosuffisant $»^{36}$, il explique qu'il s'agit d' « un humanisme n'acceptant aucune fin autre que celle correspondant à l'objectif de l'épanouissement humain, et ne s'en remettant à rien qui aille au-delà de ce dernier. Ce n'était le cas pour aucune société antérieure $»^{37}$. Dans une telle société, la vision d'une vie accomplie, c'est-à-dire pleinement vécue, est différente que dans les sociétés d'avant le désenchantement du monde pour lesquelles la notion de plénitude était toujours considérée en rapport avec la foi. Olmi avoue qu'au départ de sa réflexion il y a eu une analyse rationnelle : « pour moi, tout avait une explication, Bakhita entrait dans les ordres car Dieu se substituait à son père. C'était rationnel, je raisonnais de manière très française. Puis j'ai lu le livre officiel sur elle, paru à l'époque en Italie $»^{38}$. Selon Taylor, l'homme ne s'est pourtant pas « complètement départi de la nostalgie que l'on peut éprouver à l'égard d'une forme de transcendance $\gg{ }^{39}$ et le roman d'Olmi paraît exprimer cette nostalgie à travers le personnage de Bakhita. L'une des questions fondamentales de ce roman est : qu'est-ce que la beauté et la force d'un être ? Comment vivre sa vie avec dignité ? Le fait de poser ce genre de questions essentielles (même parfois sans espoir de trouver de réponses) exprime en même temps la foi dans la force et le potentiel éthique de la littérature. Véronique Olmi admet, elle-même, que " la littérature, ça questionne le bien et le mal $»^{40}$. Son intuition d'examiner et de narrer la réalité intérieure du personnage trouve sa formule théorique dans la conception du lieu de plénitude forgée par Charles Taylor et évoquée au début de l'article.

Reste la question de différents chemins qui peuvent conduire l'homme à cette plénitude et dont l'un d'eux est représenté par le personnage crée par Olmi qui agit « avec intégrité, générosité, abandon ou oubli de soi » et ravive la foi en l'humain.

Olmi n'est pas seule à puiser dans les biographies des saints pour interroger le côté spirituel de l'homme d'aujourd'hui. La littérature française des deux dernières décennies manifeste un certain intérêt aussi bien pour les figures des saints et des personnages bibliques ${ }^{41}$ (Hildegarde, Moïse, Tobie) que pour le cheminement intérieur de l'homme contemporain ${ }^{42}$ comme si l'« humanisme autosuffisant » et le pur rationalisme ne satisfaisaient pas à des doutes et questions concernant la souffrance, le mal, la mort, la beauté, l'amour d'autrui. Ce questionnement, actuel selon l'écrivaine, est aussi présent dans sa réflexion sur Bakhita : «La beauté de ce

\footnotetext{
${ }^{36}$ Ch. Taylor, L'Âge séculier, op. cit., p. 42.

${ }^{37}$ Ibid.

${ }^{38}$ V. Trierweiler, "Véronique Olmi plonge », op. cit.

${ }^{39}$ Ch. Taylor, L'Âge séculier, op. cit., p. 23.

40 "Bakhita " de Véronique Olmi ou le parcours d'une esclave devenue Sainte, TV5 MONDE DIRECT, op. cit.

${ }^{41}$ Cf. L. Nobécourt, La clôture des merveilles (2013) ; S. Germain, Tobie des marais (1998) ; G. Rozier, Moïse fiction (2001).

${ }^{42} C f$. E. Carrère, Le Royaume (2014).
} 
personnage, c'est qu'elle a traversé le chaos du monde [...] et elle a su garder cette part irréductible de l'âme humaine qui va se tourner vers l'altérité et c'est un enseignement pour moi qui est actuel $»^{43}$.

Le roman d'Olmi est donc représentatif, d'abord, de la littérature biographique qui a connu un certain succès surtout au $\mathrm{XXI}^{\mathrm{e}}$ siècle. De plus, comme cela vient d'être mentionné, il s'agit de la biographie d'une sainte - de l'hagiographie, le texte continue en quelque sorte l'ancienne tradition des Vitae. Enfin, l'approche d'Olmi à son personnage fait que son roman est aussi un exemple de la tendance postséculière dans la littérature contemporaine.

\section{Conclusion}

Grâce au recours aux moyens stylistiques, syntaxiques ou lexicaux, Véronique Olmi a réussi à représenter le mépris en réfléchissant sur les mécanismes de comportement humain qu'il génère. Cette réflexion l'a conduite d'un côté à la mise en doute de la nature humaine, mais de l'autre, à interroger et valoriser l'exceptionnelle humanité de Bakhita. Les descriptions détaillées des humiliations et du mépris servent, en effet, à introduire le contraste entre la réalité brutale et l'intériorité du personnage, à montrer le paradoxe de ce personnage : la bonté comme réponse à la cruauté, la soumission aux maîtres et l'insoumission intérieure au mal ambiant, la sensibilité et la force de l'âme. La narration insiste sur la dignité de ce personnage.

Est-ce qu'Olmi a répondu aux questions qui ont inspiré l'écriture de ce roman? Sûrement, elle a réussi à montrer la force et la beauté intérieure de Bakhita. Mais l'écrivaine a eu aussi la délicatesse ou l'humilité de ne pas avancer des thèses sûres là où il n'y a de place que pour le mystère. Elle affirme, elle-même que « [c]ette résistance intérieure est un mystère, Bakhita reste un mystère. Elle est beaucoup plus grande que ce que j'ai pu approcher ${ }^{44}$. Vu cela, le roman n'est probablement que l'expression de l'admiration pour Bakhita, il ne prétend pas à expliquer ce qui dépasse probablement la capacité de compréhension rationnelle.

\section{Bibliographie}

Bieńkowski, Ludomir et al. (dir.), Encyklopedia katolicka, vol. V, Lublin, Katolicki Uniwersytet Lubelski, 1989

Carreau, Nicolas et G. P., Prix Goncourt : pourquoi notre spécialiste voit 'Bakhita', de Véronique Olmi, gagner; URL: http://www.europe1.fr/culture/prix-goncourt-voici-notre-favori-3482600 ; consulté le 2.09.2018

Hurston, Zora Neale, Barracoon: The Story of the Last 'Black Cargo', Amistad Press, New York, 2018

${ }^{43}$ La Grande Librairie, op. cit.

${ }^{44}$ V. Trierweiler, «Véronique Olmi plonge... », op. cit. 
Jean-Paul II, Lettre Encyclique Fides et ratio ; URL : https://www.eleves.ens.fr/aumonerie/talatex/ fides_ratio/tex/fides_ratio.pdf; consulté le 10.04.2019

Lewis, Michael; Haviland-Jones, Jeannette M., Psychologia emocji, trad. M. Kacmajor et al., Gdańsk, Gdańskie Wydawnictwo Psychologiczne, 2005

Olmi, Véronique, Le Premier amour, Paris, Grasset, 2010

Olmi, Véronique, Czekam na Ciebie, trad. Agata Sylwestrzak-Wszelaki, Kraków, Wydawnictwo Otwarte, 2011

Olmi, Véronique, Bakhita, Paris, Albin Michel, 2017

Olmi, Véronique, Bakhita, trad. Katarzyna Marczewska, Kraków, Wydawnictwo Literackie, 2018

Le Nouveau Petit Robert, sous la dir. de Rey-Debove, Josette, Paris, Dictionnaires Le Robert

Roullet, Hervé, Bakhita, l'esclave devenue sainte, Paris, Éditions de l'Emmanuel, 2015

Roullet, Hervé, Joséphine Bakhita. De l'esclavage à la sainteté, Paris, Le Livre Ouvert, Coll. « Paroles de vie », 2019

Taylor, Charles, L'Âge séculier, trad. P. Savidan, Paris, Seuil, 2011

Taylor, Charles, La Diversité de l'expérience religieuse aujourd'hui. William James revisité, trad. Jean-Antonin Billard, Québec, Éditions Bellarmin 2003

Trierweiler, Valérie, «Véronique Olmi plonge dans le mystère. Bakhita », Paris Match, 03/09/2017; URL : https://www.parismatch.com/Culture/Livres/Veronique-Olmi-plonge-dans-le-mystereBakhita-1339076 ; consulté le 2.09.2018

Zanolini, Ida, Storia meravigliosa della vita: santa Giuseppina Bakhita, Strasbourg, Éditions du Signe, 2000

Dictionnaires biographiques des chrétiens d'Afrique; URL : https://dacb.org/fr/stories/sudan/bakhitajosephine2/; consulté le 2.09.2018

«Bakhita » de Véronique Olmi ou le parcours d'une esclave devenue Sainte, TV5 MONDE DIRECT ; URL : https://www.youtube.com/watch?v=aLk7YKAhcI0 ; consulté le 2.09.2018

La Grande Librairie : « Bakhita » racontée par Véronique Olmi ; URL : https://www.youtube.com/ watch? $=6$ A_EdcyHNbA; consulté le 2.09.2018

La confiance est le meilleur vaccin contre le mépris ; URL : https://nospensees.fr/confiance-meilleurvaccin-contre-mepris/ ; consulté le 25.09.2018

«Le clash culture : faut-il lire Bakhita de Véronique Olmi ? », Le Figaro.fr 02/12/2017 ; URL : http:// www.lefigaro.fr/livres/2017/12/02/03005-20171202ARTFIG00012-le-clash-culture-faut-il-lirebakhita-de-veronique-olmi.php ; consulté le 25.09.2018

Anna Żurawska : maître de conférences à la Chaire de Philologie romane de l'Université Nicolas Copernic de Toruń, en Pologne. Boursière du programme Comprendre le Canada et membre de l'Association Polonaise d'Études Canadiennes, elle s'intéresse à la littérature et à la culture du Québec. Elle est également l'auteure de divers articles publiés, entre autres, dans TransCanadiana, Romanica Silesiana et Quêtes littéraires ainsi que la corédactrice de l'ouvrage Homo Spiritualis aux $X X^{\mathrm{e}}$ et XXI siècles (2016) et du numéro 9 de la revue TransCanadiana (2017). Ses recherches actuelles se concentrent autour de la question du (post)sécularisme dans la littérature française et québécoise du XXI ${ }^{\mathrm{e}}$ siècle.

\begin{tabular}{|l|l|}
\hline cc) Creative & $\begin{array}{l}\text { C by the author, licensee Łódź University - Łódź University Press, } \\
\text { Łódź, Poland. This article is an open access article distributed under } \\
\text { the terms and conditions of the Creative Commons Attribution license } \\
\text { CC-BY-NC-ND 4.0 (https://creativecommons.org/licenses/by-nc-nd/4.0/) }\end{array}$ \\
\cline { 2 - 2 } & Received: 2019-01-15; Accepted: 2020-11-30 \\
\hline
\end{tabular}

